\title{
Prevalence of Class 1 Integron Among Multidrug-Resistant Acinetobacter baumannii in Tabriz, Northwest of Iran
}

\author{
AMIR PEYMANI ${ }^{1}$, SAFAR FARAJNIA ${ }^{2,3 *}$, MOHAMMAD REZA NAHAEI ${ }^{4}$, NASROLLAH SOHRABI ${ }^{5}$, LALEH \\ ABBASI $^{1}$, KHALIL ANSARIN ${ }^{2}$ and FATEMEH AZHARI ${ }^{3}$ \\ ${ }^{1}$ Department of Microbiology, Faculty of Medicine, Qazvin University of Medical Sciences, Qazvin, Iran \\ ${ }^{2}$ Tuberculosis and Lung Disease Research Center, Tabriz University of Medical Sciences, Tabriz, Iran \\ ${ }^{3}$ Biotechnology Research Center, Tabriz University of Medical Sciences, Tabriz, Iran \\ ${ }^{4}$ Drug Applied Research Center, Tabriz University of Medical Sciences, Tabriz, Iran \\ ${ }^{5}$ Department of Microbiology, Faculty of Medicine, Kermanshah University of Medical Sciences, Kermanshah, Iran
}

Received 13 October 2011, revised 8 February 2012, accepted 9 February 2012

\author{
Abstract
}

\begin{abstract}
Integrons are associated with a variety of gene cassettes, which confer resistance to multiple classes of antibacterial drugs. In this study we tested the frequency of class 1 and 2 integrons among multidrug-resistant Acinetobacter baumannii (MDRAB) clinical isolates. One hundred clinical isolates of A. baumannii were screened for carriage of class 1 and 2 integrons by PCR method. Results showed that seventy four (92.5\%) of 80 MDRAB carried class 1 integron. Integron-positive isolates were statistically more resistant to aminoglycoside, quinolone and beta-lactam compounds except for cefepime. This is the first report of class 1 integrons in MDRAB isolates in northwest Iran.
\end{abstract}

Key words: Acinetobacter baumannii, class 1 integron, multidrug resistance

Acinetobacter baumannii (A. baumannii) is one of the most important nosocomial pathogens that causes various types of clinical infections (Dinc et al., 2010). This organism is ranked second after Pseudomonas aeruginosa among gram-negative nosocomial pathogens (Berlau et al., 1999). A. baumannii is becoming a serious clinical concern due to its noticeable ability to develop resistance to several classes of antimicrobial drugs. There are increasing reports of multidrugresistant A. baumannii (MDRAB) outbreaks in clinical settings worldwide (Peleg et al., 2008). The MDRAB term was defined as those isolates that are intermediate or resistant to at least three different classes of antimicrobial agents mainly beta-lactams, aminoglycosides, fluoroquinolones and carbapenems (Huang et al., 2008). Carbapenems are currently the drugs of choice for treatment of severe infections caused by this organism. However, resistance to carbapenems in this species is now reported increasingly throughout the world (Marais et al., 2004; Merkier et al., 2008; Dijkshoorn etal., 2007). Studies have shown that MDRAB may be developing through the acquisition or horizontal transfer of the antibiotic resistance genes. Integrons are mobile genetic elements capable to cap- ture and speared of antimicrobial resistance genes. To date, several classes of integrons have been described among them class 1 and 2 integrons are the most frequently identified integrones in clinical isolates of A. baumannii that confer resistance to multiple classes of antimicrobial drugs (Oh et al., 2002). The aims of this study were to determine the frequency of class 1 and 2 integrons among MDRAB clinical isolates and to investigate the association between the presence of integrons and antibiotic resistance patterns in MDRAB isolates.

This study was carried out at a 1000 beds tertiary care hospital in Tabriz, Northwest Iran. Written informed consent was obtained from all subjects enrolled in this study and the protocol was approved by the Regional Ethical Committee. A total of 100 non-duplicated clinical isolates were collected during a period of 16 months between March 2008 and June 2009 from patients admitted to hospital. All isolates were identified using standard bacteriologic and biochemical methods such as: gram stain, oxidase test, motility, catalase test, citrate test, $\mathrm{O} / \mathrm{F}$ (Oxidation-Fermentation) test and growth at $37^{\circ} \mathrm{C}$ and $44^{\circ} \mathrm{C}$ (Feizabadi et al., 2008). Species identification was confirmed by detection of bla $a_{\text {OXA-51-like }}$ gene as described previously (Turton et al., 2006).

\footnotetext{
* Corresponding author: S. Farajnia, Biotechnology Research Center, Tabriz University of Medical Sciences, Golgasht Ave., Tabriz, Iran; phone: 00989143018589; fax: 00984113363231; e-mail: farajnias@tbzmed.ac.ir
} 
Antimicrobial susceptibility test was performed using the standard Kirby-Bauer disc diffusion method according to Clinical and Laboratory Standards Institute (CLSI) guideline. The antimicrobial agents tested were ampicillin-sulbactam $(10 / 10 \mu \mathrm{g})$, piperacillintazobactam $(100 / 10 \mu \mathrm{g})$, amoxicillin/clavulanic acid $(20 / 10 \mu \mathrm{g})$, ticarcillin/clavulanic acid $(75 / 10 \mu \mathrm{g})$, ceftriaxone $(30 \mu \mathrm{g})$, cefepime $(30 \mu \mathrm{g})$, ceftazidime $(30 \mu \mathrm{g})$, piperacillin $(100 \mu \mathrm{g})$, aztreonam $(30 \mu \mathrm{g})$, imipenem $(10 \mu \mathrm{g})$, meropenem $(10 \mu \mathrm{g})$, gentamicin $(10 \mu \mathrm{g})$, ciprofloxacin $(5 \mu \mathrm{g})$, levofloxacin $(5 \mu \mathrm{g})$, amikacin $(30 \mu \mathrm{g})$ and trimethoprim/sulfamethoxazole $(25 \mu \mathrm{g})$. All antibiotics disks were purchased from Mast, UK. The Minimum Inhibitory Concentration (MICs) of imipenem and meropenem were determined by the E-test method (AB Biodisk, Solna, Sweden) according to the CLSI guidelines. Pseudomonas aeruginosa ATCC 27853 was used as the control strain in antimicrobial susceptibility testing. Extraction of genomic DNA from A. baumannii isolates was performed by boiling method. PCR assay was performed for detection of class 1 and 2 integron genes using the following consensus primer pairs: Int1-F: 5'-GGTGTGGCGGGCTTCGTG-3', Int1-R: 5'-GCATCCTCGGTTTTCTGG-3', Int2-F 5'-CAC GGATATGCGACAAAAAGGT-3' and Int2R 5'-GTAGCAAACGAGTGACGAAATG-3' (Shibata et al., 2003). The primers were purchased from MWG Company, Germany. P. aeruginosa ATCC 27853 was used as the negative control and $P$. aeruginosa harboring integrons class 1 and 2 genes (kindly provided from Dr Shahcheraghi, Iran) were used as positive controls. Descriptive statistics including frequencies, crosstabulation of microbiological, clinical and demographic characteristics were performed using the software program SPSS, version 16 (SPSS). The chi-square test was used to determine the association between integron carriage and antimicrobial susceptibility patterns. A $P$ value of less than 0.05 was considered statistically significant.

A total of 100 clinical isolates were collected from patients admitted to different wards of Imam Reza hospital of Tabriz, Iran. The isolates were obtained from different clinical specimens including trachea (37\%), urine $(21 \%)$, sputum $(9 \%)$, blood $(7 \%)$, catheter $(6 \%)$, bronchial washings (6\%), wound (5\%), abscess drainage (3\%), cerebrospinal fluid (2\%), ascit fluid (2\%) and pleural effusion (2\%). The isolates were obtained from patients in intensive care units (38\%), internal (24\%), neurosurgery (17\%), infectious (9\%), neurology (8\%) and general surgery (3\%) wards. Seventy two patients (72\%) were males and $28(28 \%)$ were females. Their ages ranged from 14 to 86 years with a mean of 51 years. Analysis for presence of $b l a_{\text {OxA-51-like }}$ gene revealed that all isolates were positive for $b l a_{\text {OxA-51-like }}$ gene that confirmed their identity as A.baumannii. Among one hundred isolates that studied, 80 isolates exhibited the

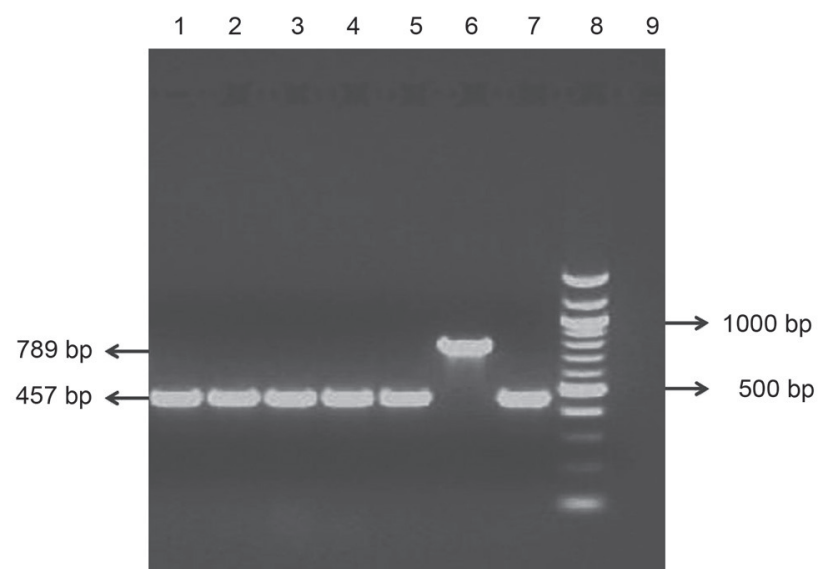

Fig. 1. PCR amplification of class 1 and 2 integron genes among clinical isolates of $A$. baumannii isolated from Imam Reza hospital of Tabriz, Iran

Lane 1-5: Positive clinical A.baumannii isolates for class 1 integron; Lane 6: Positive control for class 2 integron; Lane 7: Positive control for class 1 integron; Lane 8: 100 bp molecular size marker; Lane 9: Negative control

MDR pattern. Seventy four (92.5\%) of MDR isolates were found to have the class 1 integron, whereas only 6 isolates $(7.5 \%)$ containing class 1 integron were nonMDR. Class 2 integron gene was not found in this study (Fig. 1). Determination of MICs against imipenem and meropenem by E-test method showed that mean MICs among the MDR isolates were $26.11 \mathrm{mg} / \mathrm{l}$ and $29.88 \mathrm{mg} / \mathrm{l}$ while this value for non-MDR isolates were $1.85 \mathrm{mg} / \mathrm{l}$ and $2.12 \mathrm{mg} / \mathrm{l}$, respectively. The antimicrobial susceptibility of integron-positive and integron-negative isolates and the association between the presence of class 1 integron and antibiotic resistance patterns are shown in Table I. Among 80 class 1 integron-positive isolates, $56(70 \%)$ and $54(67.5 \%)$ isolates were meropenem and imipenem resistant, respectively. Analysis of data revealed a significant association between MDR pattern and presence of class 1 integron $(\mathrm{p}<0.001)$. The results also showed that integron-positive isolates were statistically more resistant to aminoglycoside, quinolone and beta-lactam compounds, except for cefepime. There was no association between the presence of class 1 integron and resistance to ampicilin and cefpodoxime, to which all isolates were fully resistant.

The present study showed that class 1 integron is widely distributed among MDRAB isolates in our hospital. This rate (92.5\%) is considerably higher than the rates found in other geographical regains including European countries (43\%) (Martinez-Freijo et al., 1998), United Kingdom (60\%) (Turton et al., 2005) and China (51.9\%) (Gu et al., 2007). Our study also revealed that class 1 integron was significantly associated with resistance to given antibiotics, including aminoglycosides, quinolones, and beta-lactam compounds. This is not surprising, since class 1 integron carry many antibiotic resistance gene cassettes which 
Table I

Antibiotic susceptibility profile of integron-positive and integron-negative A. baumannii isolates from Imam Reza hospital, Tabriz, Iran

\begin{tabular}{|l|c|c|c|c|c|c|c|c|c|c|}
\hline \multirow{2}{*}{$\begin{array}{c}\text { Anti- } \\
\text { biotics }\end{array}$} & \multicolumn{2}{|c|}{ Antibiotic susceptibility } & \multicolumn{2}{c|}{ Integron-positive isolates } & \multicolumn{2}{c|}{ Integron-negative isolates } & \multirow{2}{*}{$P$ value } \\
\cline { 2 - 12 } & $\mathrm{R}(\%)$ & $\mathrm{I}(\%)$ & $\mathrm{S}(\%)$ & $\mathrm{R}(\%)$ & $\mathrm{I}(\%)$ & $\mathrm{S}(\%)$ & $\mathrm{R}(\%)$ & $\mathrm{I}(\%)$ & $\mathrm{S}(\%)$ & 2 \\
\hline ATM & 94 & 2 & 4 & 74 & 0 & 2 & 20 & 2 & 2 & 0.017 \\
\hline CRO & 91 & 3 & 6 & 74 & 1 & 1 & 17 & 2 & 5 & $<0.001$ \\
\hline CTX & 93 & 3 & 4 & 76 & 0 & 0 & 17 & 3 & 4 & $<0.001$ \\
\hline CAZ & 92 & 3 & 5 & 73 & 1 & 2 & 19 & 2 & 3 & 0.029 \\
\hline PIP & 91 & 1 & 8 & 75 & 0 & 1 & 16 & 1 & 7 & $<0.001$ \\
\hline TZP & 88 & 3 & 9 & 74 & 1 & 1 & 14 & 2 & 8 & $<0.001$ \\
\hline AMC & 88 & 6 & 6 & 73 & 1 & 2 & 15 & 5 & 4 & $<0.001$ \\
\hline SXT & 86 & 3 & 11 & 72 & 1 & 3 & 14 & 2 & 8 & $<0.001$ \\
\hline FEB & 85 & 3 & 12 & 68 & 1 & 7 & 17 & 2 & 5 & 0.55 \\
\hline GEN & 85 & 5 & 10 & 69 & 4 & 3 & 16 & 1 & 7 & 0.002 \\
\hline CIP & 85 & 4 & 11 & 72 & 1 & 3 & 13 & 3 & 8 & $<0.001$ \\
\hline LVX & 83 & 2 & 3 & 71 & 2 & 3 & 12 & 0 & 12 & $<0.001$ \\
\hline TIM & 82 & 8 & 10 & 67 & 5 & 4 & 15 & 3 & 6 & 0.009 \\
\hline AMK & 80 & 2 & 18 & 67 & 2 & 7 & 13 & 0 & 11 & $<0.001$ \\
\hline SAM & 60 & 21 & 19 & 51 & 18 & 7 & 9 & 3 & 12 & 0.001 \\
\hline MEM & 56 & 6 & 38 & 50 & 6 & 20 & 6 & 0 & 18 & $<0.001$ \\
\hline IPM & 53 & 6 & 41 & 49 & 5 & 22 & 4 & 1 & 19 & $<0.001$ \\
\hline
\end{tabular}

Abbreviations: R - Resistance; I - Intermediate; S - Sensitive; ATM - Aztreonam; CRO - Ceftriaxone; CTX - Cefotaxime; CAZ - Ceftazidime; PIP - Piperacillin; TZP - Piperacillin-tazobactam; AMC - Amoxicillin-clavulanic acid; SXT - Trimethoprim-sulfamethoxazole; FEP - cefepime; GEN - Gentamicin; CIP - ciprofloxacin; LVX - Levofloxacin; TIM - Ticarcillin-clavulanic acid; AMK - Amikacin; SAM - Ampicillin-sulbactam; MEM - Meropenem; IPM - Imipenem

encode resistance to a wide range of antibiotics in Acinetobacter spp. (Perez et al., 2007). However, 7.5\% of integron-negative isolates were also resistant to these drugs. The antibiotic resistance genes of these isolates could be acquired by chromosomal-encoded enzymes or other mobile elements.

Most of integron positive isolates were resistant to aminoglycoside compounds. This could be explained by the fact that many gene cassettes conferring resistance to aminoglycosides (aad and aac genes) are carried on class 1 integron structures (Nemec et al., 2004). This study also showed the high resistance rate to several classes of beta-lactam compounds in integron-positive strains indicating carriage of several beta-lactamase encoding genes by class 1 integron. More importantly, TEM- or SHV- and OXA-types carbapenemase and metallo-beta-lactamase enzymes might have contributed in resistance to carbapaenems, extended expectrum cephalosporins, most penicillins and penicillin-beta-lactamase inhibitor compounds (Liu et al., 2006). However, we found no association between integron presence and nonsusceptibility to cefepime in MDRAB isolates suggesting that other antibiotic resistance mechanisms may be involved in resistance to this drug in our strains.

This study revealed a significant association between the presence of the class 1 integron and quinolone resis- tance. This is perhaps surprising since resistance to quinolone compounds is often results from chromosomal point mutations. However, it has been reported that integrons or associated plasmids may harbor genes encoding proteins that affect the permeability of cells or the efflux systems conferring resistance to some quinolone and betalactam compounds (Vila et al., 2007). In conclusion, this study showed high prevalence of class 1 integron among A. baumannii isolated from our hospital settings. Considering the significant association between integron carriage and reduced susceptibility to variety of antibiotics, use of appropriate infection control strategy and a regular surveillance system is necessary in our hospital. Nevertheless, further studies need to be carried out to characterize different resistance gene cassettes within integron class 1 in these isolates.

\section{Acknowledgments}

This study was supported by the Drug Applied Research Center (grant number 87/48), Tabriz University of Medical Sciences, Tabriz, Iran.

\section{Literature}

Berlau J., H.M. Aucken, H. Malnick and T.L. Pitt. 1999. Distribution of Acinetobacter spp. on skin of healthy humans. Eur. J. Clin. Microbiol. Infect. Dis. 18: 179-183. 
Clinical and Laboratory Standard Institute. 2006. Performance standards for antimicrobial disk susceptibility tests. Approved standard M2-A9. Wayne, PA: CLSI

Dijkshoorn L., A. Nemec and H. Seifert. 2007. An increasing threat in hospitals: multidrug-resistant Acinetobacter baumannii. Nat. Rev. Microbiol. 5: 939-951.

Dinc U., G. Bayramoglu, K. Buruk, H. Ulusoy, I. Tosun and N. Kaklikkaya. 2010. Molecular epidemiology of Acinetobacter baumannii-Acinetobacter calcoaceticus complex isolated from clinical specimens at an intensive care unit. Saudi. Med. J. 31: 453-455. Feizabadi MM., B. Fatollahzadeh, M. Taherikalani, M. Rasoolinejad, N. Sadeghifard, M. Aligholi, S. Soroush and S. MohammadiYegane. 2008. Antimicrobial susceptibility patterns and distribution of $b l a_{\text {OXA }}$ genes among Acinetobacter spp. Isolated from patients at Tehran hospitals. Jpn. J. Infect. Dis. 61: 274-278.

Gu B., M. Tong, W. Zhao, G. Liu, M. Ning, S. Pan and W. Zhao. 2007. Prevalence and characterization of class I integrons among Pseudomonas aeruginosa and Acinetobacter baumannii isolates from patients in Nanjing, China. J. Clin. Microbiol. 45: 241-243.

Huang L.Y., T.L. Chen, P.L. Lu, C.A. Tsai, W.L. Cho, F.Y. Chang, C.P. Fung and L.K. Siu. 2008. Dissemination of multidrug-resistant, class 1 integron-carrying Acinetobacter baumannii isolates in Taiwan. Clin. Microbiol. Infect. 14: 1010-1019.

Liu S.Y., J.Y. Lin, C. Chu, S.u. LH, T.Y. Lin and C.H. Chiu. 2006. Integron-associated imipenem resistance in Acinetobacter baumannii isolated from a regional hospital in Taiwan. Int. J. Antimicrob. Agents. 27: 81-84.

Marais E., G. de. Jung, V. Ferraz, B. Maloba and A.G. Dusé. 2004. Interhospital transfer of pan-resistant Acinetobacter strains in Johannesburg, South Africa. Am. J. Infect. Control. 32: 278-281.

Martinez-Freijo P., A.C. Fluit, F.J. Schmitz, V.S. Grek, J. Verhoef and M.E. Jones. 1998. Class I integrons in Gram-negative isolates from different European hospitals and association with decreased susceptibility to multiple antibiotic compounds. J. Antimicrob. Chemother. 42: 689-696.
Merkier A.K., M. Catalano, M.S. Ramírez, C. Quiroga, B. Orman, L. Ratier, A. Famiglietti, C. Vay, A. Di Martino, S. Kaufman and D. Centrón. 2008. Polyclonal spread of $b l a_{(\mathrm{OXA}-23)}$ and $b l a_{\text {(OXA-58) }}$ in Acinetobacter baumannii isolates from Argentina. J. Infect. Dev. Ctries. 2: 235-240.

Nemec A., L. Dolzani, S. Brisse, P. Van den Broek and L. Dijkshoorn. 2004. Diversity of aminoglycoside-resistance genes and their association with class 1 integrons among strains of panEuropean Acinetobacter baumannii clones. J. Med. Microbiol. 53: 1233-1240.

Oh J.Y., K.S. Kim, Y.W. Jeong, J.W. Cho, J.C. Park and J.C. Lee. 2002. Epidemiological typing and prevalence of integrons in multiresistant Acinetobacter strains. APMIS. 110: 247-252.

Peleg A.Y., H. Seifert, D.L. Paterson. 2008. Acinetobacter baumannii: emergence of a successful pathogen. Clin. Microbiol. Rev. 21: 538-582.

Perez F., A.M. Hujer, K.M. Hujer, B.K. Decker, P.N. Rather and R.A. Bonomo. 2007. Global challenge of multidrug-resistant Acinetobacter baumannii. Antimicrob. Agents. Chemother. 51: 3471-3484.

Shibata N., Y. Doi, K. Yamane, T. Yagi, H. Kurokawa, K. Shibayama, H. Kato, K. Kai and Y. Arakawa. 2003. PCR typing of genetic determinants for metallo-beta-lactamases and integrases carried by gram-negative bacteria isolated in Japan, with focus on the class 3 integron. J. Clin. Microbiol. 41: 5407-5413.

Turton J.F., M.E. Kaufmann, J. Glover, J.M. Coelho, M. Warner, R. Pike, T.L. Pitt. 2005. Detection and typing of integrons in epidemic strains of Acinetobacter baumannii found in the United Kingdom. J. Clin. Microbiol. 43: 3074-3082.

Turton J.F., N. Woodford, J. Glover, S. Yarde, M.E. Kaufmann and T.L. Pitt. 2006. Identification of Acinetobacter baumannii by detection of the bla $a_{\text {OXA-51-like }}$ carbapenemase gene intrinsic to this species. J. Clin. Microbiol. 44: 2974-2976.

Vila J., S. Martí and J. Sánchez-Céspedes. 2007. Porins, efflux pumps and multidrug resistance in Acinetobacter baumannii. J. Antimicrob. Chemother. 59: 1210-1215. 